\title{
JUSTUS LIEBIG'S \\ ANNALEN DER CHEMIE.
}

427. Band.

[Mitteilungen aus dem Chemischen Laboratorium der Universitat: Freiburg i. Br.]

Öber Dicyanamid;

von W. Madelung and E. Kern.)

(Eingelaufen am 31. Oktober 1920.)

Die im folgenden beschriebenen Untersuchungen bezwecken einen Beitrag zu bilden zur Beantwortung der Frage nach dem Zusammenhang zwischen chemischer Konstitution und elektrochemischen Eigenschaften der Verbindungen. Seit den klassischen Untersuchungen Ostwalds über die Affinitätsgröße organischer Säuren sind eine grobe Zahl von Untersuchungen über dieses Thema erschienen, die Fülle beigebrachten Naterials hat jedoch bisher wohl eine Reihe von Znsammenhängen anfgedeckt, aber noch nicht zur Aufstellung einer zureichenden, weil allgemein gültigen, Hypothese geführt, die die besonderen elektrochemischen Eigenschaften anorganischer wie organischer Verbindungen einheitlich als Funktion bestimmter konstitutioneller Eigenschaften erkennen lassen. Allgemein gültige Schlüsse lassen sich nun in erster Linie dann ziehen, wenn man die Wirkung bestimmter Gruppen mit besonders ausgeprägten reaktivierenden Eigenschaften in einer größeren Zahl von Verbindungen möglichst einfacher Zusammensetzung miteinander vergleicht.

1) Vgl. die gleichnamige Inauguraldissertation von E. Kern, Freiburg i. Br. 1919. 
Für derartige Untersuchungen scheinen die Cyanverbindungen besonders geeignet zu sein, weil einerseits die Cyangruppe eine der wirksamsten reaktiven Gruppen ist, die wir kennen, sie andererseits aber infolge ihrer Reaktionsfähigkeit leicht in die verschiedensten Umwandlungsprodukte von z. T. ebenfalls ausgeprägten elektrochemischen Eigenschaften verwandelt wird. Eine genaue Kenntnis der Cyanverbindungen des Ammoniaks schien uns besonders erwiinscht, ist doch das Ammoniak und die Aminogruppe die Atomkombination, die die stärkste reaktivierende Wirkung im umgekehrten Sinne wie die Cyangruppe zeigt. Das Cyanamid kann man daher als den Prototyp einer Verbindung betrachten, in der negative and positive Gruppen miteinander vereinigt sind, und sich daher die Wirkung der einzelnen Gruppen gewissermaßen gegenseitig aufhebt. Es erschien uns daher als Aufgabe von hohem Interesse zu untersuchen, welche Erscheinungen auftreten, wenn in die Molekel des Cyanamids ein zweiter: Cyanrest eintritt.

Das Dicyanamid, $\mathrm{NH}(\mathrm{CN})_{2}$, ist trotz seiner einfachen Zusammensetzung bis jetzt noch unbekannt. Versuche zu seiner Darstellung sind beschrieben worden, die aber nicht znm Ziele führten. So lieben \%ech und Dehmel ${ }^{1}$ ) auf freies Cyanamid Bromcyan einwirken in der Meinung, dieses müsse in der gleichen Weise wio bei seinem ersten Einwirken auf Ammoniak, ein zweites Mal reagieren. Sie erhielten das Bromcyan unverändert zurück, während sich das Cyanamid polymerisierte.

Fine Verbindung von der Zusammensetzung des Dicyanamids glaubte Bannow ${ }^{2}$ ) beobachtet zu haben. Fr gibt an, beim Zusammenschmelzen von Kaliumcyanid mit Paracyan bzw. Quecksilbercyanid und Frhitzen auf Rotglut geringe Mengen einer in Nadeln krystallisierenden Verbindung erhalten zu haben, die er für eine solche der

1) Ber. d. d. chem. Ges. 11, 249 (1878).

\% Ber. d. d. chem. Ges. 4, 254 (1871); 13, 2201 (1880), 
Zusammensetzung $\mathrm{C}_{2} \mathrm{~N}_{3} \mathrm{~K}$ hielt, da er daraus mit Silbernitrat eine auf $\mathrm{C}_{2} \mathrm{~N}_{3} \mathrm{Ag}$ stimmende Silberverbindung erhielt. Die von Bannow erhaltenen salzartigen Verbindungen sind aber nicht solche des Dicyanamids, sondern, wie im folgenden gezeigt werden wird, Polymere desselben.

Die Eigenschaften des Dicyanamids und damit der geeignetste Weg zu seiner Darstellung ließen sich bis zo einem gewissen Grade voraussehen. IIir wissen aus zahlreichen Beobachtnngen, daß sich durch mehrfache Einführung von im gleichen Sinne reaktivierenden Gruppen sanre oder basische ligenschaften sowohl hervorrufen wie auch, wenn schon vorhanden, enorm steigern lassen. Wir wollen hier nur die Verbindungen ins Auge fassen, die durch Eintritt einer oder mehrerer Cyangruppen in die Wasserst offverbindungen derMetalloide entstehen.

\begin{tabular}{l|l|l|c}
\hline \hline $\mathrm{CH}_{4}$ & $-\ldots$ & $\ldots \ldots$ & - \\
$\mathrm{CH}_{3} \mathrm{Cy}$ & $\mathrm{NH}_{3}$ & - & $\ldots$ \\
$\mathrm{CH}_{z} \mathrm{Cy}_{2}$ & $\mathrm{NH}_{2} \mathrm{Cy}$ & $\mathrm{SH}_{2}$ & - \\
$\mathrm{CHCy}_{3}$ & $\mathrm{NHCy}_{2}$ & $\mathrm{SHCy}$ & $\mathrm{ClH}$ \\
- & $\cdots$ & $\mathrm{SCy}_{2}$ & $\mathrm{ClCy}$
\end{tabular}

Wenn wir, wie es in obiger Tabelle geschieht, diejenigen Verbindungen in eine Reihe stellen, die neben den als reaktivierend angenommenen Cyangruppen and den an erster Stelle die "'asserstoffverbindung bildenden Atomen verschiedener Elemente stets die gleiche Zahl von Wasserstoffatomen enthalten, so bemerken wir, dab diese Verbindungen sich in ihren Kigenschaften weitgehend entsprechen. Beim Methan erliält man erst bei Eintritt zweier Cyangruppen, im Malonitril, einen ähnlichen Effekt wie beim Ammoniak beim Eintritt einer einzigen Cyangruppe, im Cyanamid, und beide Cyanverbindungen entsprechen dann in ihren Kigenschaften dem Schwefelwasserstoff; es sind Verbindungen, die alle etwa in gleichem Maße zar Salzbildung befähigt, aber doch zu 
schwach sauer sind, um in wäbriger Lösung saure Reaktion hervorzurnfen. Wenn man eine weitere Cyangruppe in diese Verbindangen einführt, so zeigt sich im Falle des Methanderivates, dab hier (im Cyanoform) eine der stärksten organischen Säuren vorliegt, die nach den Untersuchungen von Hantzsch und Osswald ${ }^{1}$ ) mit deu starken anorganischen Säuren in eine Reihe zu stellen ist, während man schon aus den Untersuchungen von O stwald ${ }^{2}$ ) vom Rhodanwasserstoff, dem cyansubstituierten Schwefelwasserstoff, weiB, daß er in seiner Stärke an den in obiger Tabelle in der glcichen Reihe stehenden Chlorwasserstoff vollkommen heranreicht. Auch die wasserstoffireien Verbindungen Chlorcyan und Schwefelcyan zeigen weitgehende Analogien in ihrem Verhalten, denen man nach den neuesten Untersuchungen von Söderbäck ${ }^{3}$ ) auch noch diejenigen zwischen freien Halogenen no freiem Rhodan zur Seite stellen könnte. Das Dicyanamid bildete nun bisher ein fehlendes Glied in dieser Reihe, und man konnte daher wohl mit einiger Wahrscheinlichkeit voraussetzen, daß seine Eigenschaften denjenigen seiner Nachbarn in der obigen Tabelle entsprechen würden. Diese Eigenschaften sind aber neben den sehr stark sauren Eigenschaften gekennzeichnet. durch eine besonders große Unbeständigkeit der freien Wasserstoffverbindungen. Während freies Cyanoform bisher überhaupt nicht isoliert werden konnte, ist Rhodanwasserstoff nur im Kältegemisch beständig und polymerisiert bei gewöhnlicher Temperatur sehr schnell. Es war daher auch für das Dicyanamid das Gegebene, nicht nach der freien Verbindung, sondern nach den beständigeren Salzen zu suchen.

Es ist uns gelungen, eine ebenso einfache wie ausgiebige Methode zur Darstellung von Natriumdicyanamid.

1) Ber. d. d. chem. Ges. 32, 641 (1899).

) Journ. f. prakt. Chem. [2] 32, 305 (1855).

3) Diese Annalen 419, 217 (1919). 
ansgehend vom technischen Dinatriumsalz des Cyanamids, zn finden. LäBt man nämlich unter Kühlung Bromcyan anf eine wäßrige Lösung dieser Verbindung einwirken, bis die zuerst alkalische Reaktion der Lösnng neutral geworden ist, so erhält man beim Eindampfen auf dem Wasserbade das Natrinmsalz des Dicyanamids in guter Ausbeute. Es krystallisiert in Nädelchen aus and läßt sich von dem gleichfalls entstehenden Natriumbromid infolge seiner geringeren Löslichkeit leicht trennen and aus Alkohol umkrystallisieren.

In hiervon abweichender Weise reagiert Cyanamid, das in absolut alkoholischer Lösung zunächst mit einer zur Bildung des Dinatriumsalzes hinreichenden Menge Natriumalkoholat und darauf, wie für die wäßrige Lösung beschrieben, mit Bromcyan versetzt wird. Diese Versuchsanordnung war übrigens die erste, da wegen der hydrolytischen Dissoziation des Dinatriumcyanamids in wäßriger Lösung Komplikationen vermutet wurden. Als Reaktionsprodukt konnte neben Natrinmdicyanamid und Natriumbromid ein weiteres Natrinmsalz von der Zusammensetzung eines alkoholhaltigen Natriumdicyanamids isoliert werden. Diese Verbindung ist jedoch nicht einfaches krystallalkohol-haltiges Natriumdicyanamid, denn letztere Verbindung wird beim Krystallisieren ans Alkohol stets alkoholfrei erhalten. Immerhin ist auch bei der alkoholhaltigen Verbindung der Alkohol nicht sehr fest gebunden, denn schon beim Umkrystallisieren wurde teilweise alkoholfreies Natriumdicyanamid zarückerhalten, und mit manchen Schwermetallsalzen, wie denen des Silbers nnd liupfers, erhält man direkt Fällungen des Dicyanamidsalzes. Trotzdem sind auch im chemischen Verhalten dentliche Unterschiede vorhanden, so reagiert die alkoholhaltige Verbindang schwach alkalisch, während das Natriumdicyanamid völlig neutrale Reaktion zeigt. Es scheint demnach ein Mol. Alkohol, wenn auch leicht abspaltbar, doch chemisch gebunden zo sein, entsprechend der Formel 


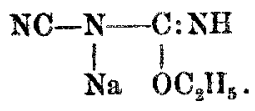

Durch eine schwache Säure wie Essigsänre läßt sich in der Tat aus dem Natriumsalz Cyan-0.äthylisoharnstoff, der Iminoäther der Cyanamidokohlensäure, eine ganz beständige, neutral reagierende Verbindung, in Freiheit. setzen, indessen erhält man diese Verbindung, wie später ausgeführt wird, anch ausgehend vom 1icyanamid.

DieUmsetzung des Natriumcyanamids mit Bromcyan in alkoholischer lösung ist ebensowenig wie die in wäßriger Lösung als eine solche des Dinatriumsalzes aufzufassen. In wäBriger Lösung ist das zweibasische Salz zweifellos größtenteils hydrolytisch gespalten, und die bei seiner Lösung in Wasser entstehende Natronlange wird dann wieder zum Neutralisieren des sich bei der Reaktion zwischen Mononatriumcyanamid und Bromcyan bildenden Dicyanamids verbraucht. Ebenso ist in der Lösung des Cyanamids in zwei Mol. Natriumalkoholat enthaltendew Alkohol nicht das Dinatriumsalz enthalten, das, sobald es einmal als fertige Verbindung vorliegt, in absolutem. Alkohol fast unlöslich ist. Nach Drechsel ${ }^{1}$ ) wird durch Umsatz ron Cyanamid mit Natriumalkoholat das Mononatrinmcyanamid erhalten, es ist aber recht wahrscheinlich, daß dieses nicht das erste Reaktionsprodukt ist, Cyanamid und Natriumalkoholat sich vielmehr zunächst miteinander vereinigen. Diese Vereinigung eatspräche der Bildung des äthylkohlensauren Natriums aus Natriumalkoholat und Kohlendioxyd, als dessen Di. imid das Cyanamid in der Isoform aufgefabt werden kann. Dieses erste Anlagerungsprodukt müßte ein Natriumsalz des 0 - ̈̈thylisoharnstoffs sein, das als solches wegen der leichten Alkoholabspaltung nicht isoliert werden kann, wohl aber zur Umsetzung mit Bromcyan befähigt wäre. Dabei entsteht dann 0-Äthylcyanisoharastoff, welche Verbindung nunmehr ebenso wie die be-

1) Journ. f. prakt. Chem. [2] 11, 307 (1875). 
kannten analog zusammengesetzten Acetyl- und Benzoylderivate des 0 -Methylisoharnstoffs ${ }^{1}$ ) beständige Metallsalze bilden kann. Bei Dialkylcyanamiden ${ }^{2}$ ) ist übrigens die Isoharnstoffätherbildung durch Einwirkung von Natriumalkoholat auf jene bekannt, die hier auch als Darstellungsmethode dienen kann, da die Bildnng eines Cyanamidsalzes nicht möglich ist.

Nachdem es möglich war, das Natriumsaly des Dieyanamids jederzeit in beliebiger Nenge darzustellen, mußte es besonderes Interesse erregren, die so einfach zusammengesetzte Verbindung $\mathrm{C}_{2} \mathrm{~N}_{3} \mathrm{H}$ selber darzustellen and ihre Figenschaften kennen zu Jernen. Aus den Eigenschaften des Natriumsalzes, das eine völlig neutral reagierende Verbindung ist, war bereits $/$ n entnehmen, daß das Dicyanamid eine starke Süure sein mïsse. Des weiteren fand aber auch der Analogieschlub aus dem Verhalten des Cyanoforms und des Rhodanwasserstoffs seine volle Bestätigung. Freies Dicyanamid ist so unbeständig and neigt so sehr zur Polymerisation, dab es uns trotz langer Bemühungen nirht möglich war, die freie Verbindung in reinem Zustande zu fassen. Mit Hilfe von Leitfähigkeitsmessungen gelang es uns trotzdem, über die Stärke der Säure ein ganz genaues Bild zu erhalten.

Wir verfuhren zu diesem $Z$ wecke in der Weise, daB wir die Lösung des Natrinmsalzes mit einer genau berechneten Menge titrierter Salzsäure versetzten und die Leitfähigkeit des so erhaltenen Gemisches von Kochsalz und freiem Dicyanamid bei verschiedenen Verdünnungen bestimmten. Durch Abzug der bekannten Leitfähigkeit des Kochsalzes ergab sich die gesuchte des freien Dicyanamids. Der Grenzwert der Leifähigkeit wurde in der bekannten von Bredig angegebenen Weise durch

1) Bruce, Journ. Am. ehom soc. 26, 419 (1904).

) Stieglitz u, Mc Kee, Ber. d. d. chem. Ges. 33, 807 (1900); Me Kee, Am. chem. Journ. 42, 1 (1909). 
Extrapolation ans der Leitfähigkeit verschieden verdủnnter Lösungen des Natriumsalzes ermittelt.

Wie die Untersuchungen zeigen, gehört Dicyanamid zu den stärksten organischen Säuren und kommt der Salzsäure in ihrer Stärke sehr nahe. Das geht schon daraus hervor, daß die Leitfähigkeiten bei mäßigen Ver dünnungen dem Grenzwerte ungefähr eben so nahe sind wie die entsprechenden der Salzsäure. Zum Vergleiche diene folgende Tabelle, die eine Reihe von äquivalenten Leit fähigkeitswerten enthält, unter ihnen die der stärksten organischen und anorganischen Säuren unter gleichen Versuchsbedingungen, d. h. bei gleicher molekularer Ver dünnung und gleicher Temperatur.

$A$ für verschiedene Säuren bei $25^{\circ}$. )

\begin{tabular}{|c|c|c|c|c|c|c|}
\hline $1 / 10^{3} \eta=$ & 64 & 128 & 256 & 512 & 1024 & $\begin{array}{c}\text { Grenzwert } \\
\infty\end{array}$ \\
\hline Salzsäure & 399 & 401 & 403 & 405 & 404 & 422 \\
\hline Dicyanamid. . . . & 369 & 371 & 372 & 373 & 374 & 392 \\
\hline Rhodanwasserstoff & 386 & 391. & 391 & 393 & 394 & - \\
\hline Cyanofirm . . . . & 369 & 374 & 379 & 380 & 381 & 391 \\
\hline Nitroform . . . & 358 & 371 & 372 & 374 & - & 394 \\
\hline Trichloressigsäure & 355 & 363 & 371 & 377 & 379 & 381 \\
\hline Monochloressigsäure. & 103 & 136 & 175 & 219 & 266 & 386 \\
\hline Essigsäuxe . . & 13 & 18 & 25 & 34 & 49 & 385 \\
\hline
\end{tabular}

Die unmittelbar nach dem Zusammenbringen äquivalenter Mengen von Dicyanamidnatrium und Salzsäure gemessenen Leitfähigkeiten sinken besonders bei stärkerer Konzentration infolge der Polymerisation mit hinreichender Geschwindigkeit, am diese messend verfolgen zu können. Die für $25^{\circ}$ und 64 fache Verdünnang der Normallösung anfgenommene Kurve des Leitvermögens des Gemisches von Dicyanamid and Natriumchlorid nähert. sich nach anfangs schnellem Abfall einem zwischen dem

1) Die von Ostwald und Hantrsch für die angeführten Werte des Leitvermögens gegebenen Zahlen sind alle von Queck. silber auf $\mathrm{Ohm}^{-1} \cdot \mathrm{eem}^{-1}$ umgerechnet. 
f́nfangswert and dem für reines Natrinmchlorid anzunebmenden Wert liegenden Zwischenwert. Die Polymerisation führt demnach zunächst zu einem relativ stabilen, noch Leitvermögen besitzenden Produkt. Wenn man dann aber eindampft, so geht auch der Rest von Leitvermögen des polymerisierten Dicyanamids verloren, and man erhält beim Zusatz von Wasser zum Rückstand nur noch das Leitvermögen des Natriumchlorids, während sich das Dicyanamid vollständig in eine amorphe unlösliche Maasse verwandelt hat. Nach den Angaben zu urteilen, die für die zum Vergleich herangezogenen Verbindungen Rhodanwasserstoff und Cyanoform vorliegen, scheint von den drei unbeständigen Cyanderivaten die Beständigkeit des Dicyanamids am geringsten zu sein.

Die chemischen Eigenschaften des Dicyanamids stehen gewissermaßen zwischen denen der beiden Vergleichsverbindungen. So erinnert es, abgesehen von der großen Stärke als Säure und der leichten Polymerisierbarkeit, auch in den Eigenschaften seiner Salze an Rhodanwasserstoff, in geringerem Maße anch an die Halogenwasserstoffe. So erhält man in Wasser unlösliche, aber ammoniaklösliche, den Rhodan und Halogenverbindungen entsprechende farblose Salze des einwertigen silbers, Kupfers und Quecksilbers, schwerlösliche Salze des Bleis, des zweiwertigen Quecksilbers und Kapfers. Besonders das letytere dunkelblau-grüne $\mathrm{Salz}$ ist charakteristisch.

Anch einige sonst für Rhodanionen charakteristische Farbreaktionen mögen an dieser Stelle erwähnt werden, die man ebenso auch mit Dicyanamidsalzen erhält. So geben Ferrisalze mit Dicyanamidnatrium eine allerdings unbeständige und weniger intensive Rotfärbung, und auch mit konz. Salpetersäare erhält man eine der nach Söderbäck auf der Bildung von Nitrosylrhodunid beruhenden unbeständigen Rotfärbung analoge Reaktion.

Eine bemerkenswerte Eigenschaft vieler Salze des Dicyanamids ist die, dal sie sich beim Erhitzen z. T. 
anter Anfglühen, teilweise anch unter Verputiung zersetzen, ein Zeichen des hohen Gehaltes an chemischer Energie. Die Alkaliverbindungen gehen dabei ohne heftigere Reaktion in trimere Salze über, die dann auch noch bei Rotglut beständig sind. Von diesen soll in der nächsten Mitteilung die Rede sein.

Von besonderem Interesse ist das Ammoniumsalz. Die schön krystallisierende Verbindung schmilyt bei $116^{\circ}$ ohne Zersetzung. Man hätte erwarten können, daß sie beim weiteren Erhitzen ähnlich dem Ammoniumcyanat oder Sulfocyanat sich umlagern und Cyanguanidin (Dicyandiamid) ergeben sollte, indessen wird bei weiterem: Erhitzen von $128^{\circ}$ ab Ammoniak abgespalten, und man erhält augenscheinlich das gleiche hochmolekulare unlösliche Polymerisationsprodukt, wie direkt aus dem treien Dicyanamid.

Aus dem Silbersalz konnte, allerdings schwierig und mit geringer Ausbeute, mit Methyljodid ein Methylderivat gewonnen werden als schön krystallisierende, bei $221^{*}$ schmelzende Verbindung. Andere Umsetzungen, die versucht wurden, versagten, da die Salze des Dicyanamids lange nicht so reaktionsfähig gegen Halogenverbindungen sind wie etwa Silber- oder Quecksilbersalze des Rhodanwasserstoffs. So war es uns nicht möglich, das Silbersalz mit Methylenjodid nmzusetzen. Auch die über das Silber- und Quecksilbersalz versuchte Einführung eines dritten Cyanradikals in die Ammoniakmolekel oder die Verknüpfung zweier Dicyanstickstoffreste $z$ u einem Tetracyanhydrazin, das mit Hinblick auf das kürzlich durch Söderbäck ${ }^{1}$ ) bekannt gewordene freie Rhodan besonderes Interesse erregt hätte, konnte bisher noch nicht unzweideuig beobachtet werden.

Das Anlagerungsvermögen der beiden Cyangruppen des Dicyanamids ermöglicht es durch Aufnahme von Wasser, Ammoniak oder Alkohol za Verbindungen zu.

1) Diese Amalen 419, 217 (1919). 
gelangen, in denen je nach Art und Zahl der eintretenden Molekeln die sauere Funktion dieser stïrkst $n$ organischen Säure abgeschwächt, aufgehoben oder schließlich bis zur stärksten basischen Funktion umgewandelt wird.

Es gelingt leicht, an das Dicyanamid zwei Mol. Wasser anznlagern unter Überführung in die neutrale Verbindung Biuret, die sich alsbald ausscheidet, wenn man das Natriumsalz des Dicyanamids kurze Zeit mit heißer, starker Salzsänre behandelt. Die Stufe des Cyanharnstoff's mit nur einem angelagerten Mol. Wasser konnte unter diesen Bedingungen nicht festgehalten werden. Wir erhielten diese von Hallwachs ${ }^{1}$ ) unter dem Namen Amidodicyansäure ausfïhrlich beschriebene Verbindung bei dem Versuche, anf einem anderen Wege zam Natriumdicyanamid $/ \mathfrak{n}$ gelangen; nämlich bei der aufeinanderfolgenden Behandlung einer wäbrigen Lösung von Natriumcyanamid mit Brom und Natrinmcyanid, die nach der folgenden Reaktionsgleichung hätte verlaufen sollen:

$$
\begin{aligned}
& (\mathrm{CN})-\mathrm{N}\left\langle\frac{\mathrm{Na}}{\mathrm{Na}}+\mathrm{Br} \mathrm{r}_{2}=(\mathrm{CN})-\mathrm{N}\left\langle_{\mathrm{Na}}^{\mathrm{Br}}+\mathrm{NaBr},\right.\right. \\
& (\mathrm{CN})-\mathrm{N}\left\langle\frac{\mathrm{Br}}{\mathrm{Na}}+\mathrm{NaCN}=(\mathrm{CN})_{2}=\mathrm{N}-\mathrm{Na}+\mathrm{Nabr} .\right.
\end{aligned}
$$

Cyanharnstoff ist noch eine kräftige einbasische Säure und zeigt auch noch in manchen Salzen, wie dem Silbersalz, Ähnlichkeiten mit denen des Dicyanamids. Wenn auch ihre Dissoziationskonstante bisher nicht bestimmt ist, so läßt sich doch ans den bekannten Dissoziationskonstanten anderer substituierter Cyanamide ${ }^{2}$ ) schließen, daß es sich bei ihs um eine der stärkeren organischen Säuren handelt (Acetylcyanamid $K=0,015$, Benzoylcyanamid $K=0,18$, Cyanurethan $K=0,047$ ).

Die Anlagerung von Ammoniak an das Dieyanamid gelingt, wenn man die Lösung seines Kupfersalzes in

) Diese Annalen 153, 293 (1870).

) Bader, Zeitschr. f. physikal. Chem. 6, 289 (1890). 
wäBrigem Ammoniak mehrere Stunden im Rohr erhitzt. Wenn man das Erhitzen rechtzeitig unterbricht, so finden sich nach dem Erkalten in der Flüssigkeit farblose Krystalle, die sich als Cyanguanidin (Dicyandiamid) erwiesen, während man bei hinreichend langem Erhitzen nur noch die charakteristischen rosafarbenen Krystalle des Biguanidkapfers isolieren kann. Cyanguanidin ist eine neutrale Verbindung, während Bignanid eine änßerst starke Base ist, da in ihr zweimal die basische Funktion. des Guanidins enthalten ist, das man in der Leitfähigkeit seiner Lösungen and im Einfluß anf die Verseifungsgeschwindigkeit von Estern usw. als dem Ätznatron nahestehend betrachten kann. Wir finden so, daß vielleicht die stärkste organische Base mit der stärksten organischen Säure genetiseh verknüpft ist durch zweimalige Anlagerung einer Verbindung, die an sich nur so schwach basische Eigenschaften besitzt wie das Ammoniak.

Wie man durch Anlagerung von Ammoniak an Dicyanamid zum Cyangaanidin, dem Amidin der Cyanamidokohlensäure gelangt, so erhält man durch Anlagerung von Alkohol Cyanisoharnstoffäther, den jener Verbindung entsprechenden Iminoäther, dessen direkte Bildung aus Cyanamid, Bromcyan und Natriumalkoholat bereits erwähnt warde. Vom Natriumdicyanamid ausgehend erhält man diese Verbindung, wenn man zu einer Lösnng des Salzes in absolutem Alkohol eine genan äquivalente Menge alkoholischer Salzsäure hinzafügt. Es wird dann gerade so wie in wäßriger Lösung ein grober Teil des Dicyanamids $z u$ einem amorphen unlöslichen Körper polymerisiert, ein kleinerer Teil wandelt sich aber in 0-Äthylcyanisoharnstoff nm. Diese schön krystallisierende, völlig neutrale and in den meisten organischen Lösungsmitteln leicht lösliche Verbindung steht zum 0-Äthylisoharnstoff in demselben Verhältnis, wie das Cyanguanidin (Dicyandiamid) zum Guanidin. Wenn so durch einmalige Einführung des Cyanrestes in diese starken 
Basen an Stelle von Wasserstoff die basische Funktion derselben bis zur völlig neutralen Reaktion abgeschwächt wird, so auch umgekehrt die änßerst starke Säurefunktion des Dicyanamids dadurch, daß die eine Cyangruppe durch Anlagerung des anscheinend indifferenten Alkohols in den Iminoätherrest übergeführt wird. Es sei hier übrigens daranf hingewiesen, daß die Anlagerung von Alkohol an das freie Dicyanamid derjenigen an das freie Cyanoform genau entspricht, bei der ebenfalls ein nentral reagierender Iminoäther, der Dicyanacetiminoäther entsteht, ein weiterer Beleg für die Analngie beider Verbindungen.

Durch weitere Einwirkung überschüssigen Chlorwasserstoffs auf Dicyanamid in alkoholischer Lösung erwarteten wir beide Cyanreste in die Reste von Iminoathern überzuführen und so zum Dihydrochloride einer zweisäurigen Base zu gelangen, die zum Biuret im gleichen Verhältnis stehen müßte, wie Isoharnstoffäther zum Harnstoff. In dieser Erwartung sahen wir uns indessen getäuscht, denn das schwer lösliche Hydrochlorid, das sich binnen kurzem aus der Lösung ausschied, war das Monohydrochlorid einer Base, die sich vom Biuret dadurch ableiten läßt, daß diese Verbindung nur zur Hälfte als Iminoäther auftritt. $D a$ die erwartete Verbindnng sich zweifellos als erstes Reaktionsprodukt gebildet haben muß, ist demnach bei ihr schon gleich nach ihrer Bildung, and zwar bei Zimmertemperatur, eine Abspaltung von Chloräthyl eingetreten, eine Reaktion, die bei allen Hydrochloriden der Iminoäther als bei höherer Temperatur eintretend bekannt ist.

Die bei dem Hydrochlorid der zweisäurigen Base so auffallend leicht erfolgende Abspaltung von Chlorathyl scheint uns von theoretischem Gesichtspunkte aus recht bemerkenswert zu sein. Das nach einmaliger Chloräthylabspaltung entstehende Hydrochlorid zeigt ein ganz beständiges normales Verhalten und zerset/t sich erst beim Erhitzen auf höhere Temperatur unter Binretbildung. Die aus dem Hydrochlorid in Freiheit gesetzte 
Base zeigt, wie zu erwarten war, in wäßriger Lösung eine dem gewöhnlichen Isoharnstoffäther entsprechende, wenn anch nicht sehr starke alkalische Reaktion.

\section{Versuche.}

\section{Natriumdicyanamid.}

In eine normale wäBrige Lösung des technischen Natriumcyanamids, die eventuell noch zur Reinigung durch Kohle filtriert worden ist, trägt man unter Kühlung so lange festes Bromcyan (1 Mol) ein, bis die alkalische Reaktion verschwunden ist. Da die Reaktion unter starker Wärmeentwicklung vor sich geht, mab man für gute Kühlung Sorge tragen, da sich sonst das Bromcyan verflüchtigt, ohne in Reaktion zu treten; auch muß man, da sich die Verflüchtigung nicht ganz vermeiden läbt, einen geringen Überschub anwenden, um sicher vollständig neutrale Reaktion zu erzielen. Letzteres ist unbedingt notwendig, da man sonst beim Eindampfen keine Krystalle, sondern nur amorphe Flocken erhält, die sich bei Finwirkung von freiem, durch Hydrolyse des Natriumcyanamids entstandenen Alkali auf schon fertiges Natriumdicyanamid bilden. Nach dem Abfiltrieren von Verunreinigungen, die sich aus der neutralen oder schwach sauren Lösung ausscheiden, wird auf dem Wasserbade eingedampft. Zuerst scheidet sich das schwerer lösliche Dicyanamidsalz aus, erst bei weiterem Einengen Natriumbromid. Durch mehrmalige fraktionierte Krystallisation gelingt es leicht, das Dicyanamidnatrium vollständig frei von Natriumbromid zu erhalten. Das Rohprodukt ist stets grünlichgelb gefärbt, wird aber beim Umkrystallisieren rein weik. Da das Natriumdicyanamid in schmalen Nadeln krystallisiert, ist es leicht, unter dem Mikroskop die Reinheit von den Würfeln des Natrinmbromids zu erkennen, ebenso auch durch die rein weiße Farbe des Silbersalzes.

Natriumdicyanamid ist in Wasser leicht löslich, 
etwas weriger leicht in Alkohol, aus dem es für die Analyse umkrystallisiert warde. Man erhält es so meist in feinen weißen Nadeln. Verschiedentlich wurden auch Wiskrystallen ähnliche sechsstrahlige Gebilde beobachtet. Die wäßrige Lösung reagiert vollständig neutral gegen Tarkmus.

$0,2190 \mathrm{~g}$ gaben $0,1722 \quad \mathrm{Na}_{3} \mathrm{SO}_{4}$.

$0,0614 \mathrm{~g}, 26,0 \mathrm{ccm}$ Stickgas bei $20^{\circ}$ und $744 \mathrm{~mm}$ Druck.

$\begin{array}{ccc} & \text { Ber. für } \mathrm{C}_{3} \mathrm{~N}_{3} \mathrm{Na} & \text { Gef. } \\ \mathrm{N} & 47,19 & 47,17 \\ \mathrm{Na} & 25,89 & 25,16\end{array}$

Silberdicyanamid fällt als weißer flockiger Niederschlag aus bei Zusatz von Silbernitrat zu einer wäBrigen Lösung von Dicyanamidnatrium. Die Verbindung ist in heißem Wasser unlöslich, ebenso auch in verdünnter oder starker Salpetersäure. Sie ist leicht löslich in verdünntem Ammoniak und wird darans durch Säuren nnverändert wieder ausgefällt. In feuchtem Zustand färbt sie sich bei lïngerem Stehen am Lichte dunkel.

$0,7 \div 02 \mathrm{~g}$ gaben $0,6133 \mathrm{AgCl}$.

$$
\begin{aligned}
& \text { Ber. für } \mathrm{C}_{2} \mathrm{~N}_{3} \mathrm{Ag} \quad \text { Gef. } \\
& \text { Af } \quad 62,03 \quad 59,95
\end{aligned}
$$

Das Salz des zweiwertigen Kupfers fällt als blaugrüner Niederschlag auf Zusatz einer Cuprisalzlösung zu einer solchen von Dicyanamidnatrinm. Beim Zasammengießen heißer Lösungen erhält man es in schön ausgebildeten blaugrünen Nädelchen. In kaltem Wasser ist die Verbindung fast unlöslich, in heißem ist sie wenig löslich, ebenso in verdünnten Mineralsäuren. Leicht löslich mit tief violettblaner Farbe ist sie in Ammoniak. Beim Erhitzen auf höhere Temperatur zersetzt sich das Kupfersalz unter Verpuffung. Wegen seiner charakteristischen Eigenschaften eignet es sich besonders für den Nachweis der Ionen des Dicyanamids.

$0,2812 \mathrm{~g}$ gaben $0,1148 \mathrm{Cu}_{2} \mathrm{~S}$.

$\begin{array}{ccc} & \text { Ber. für } \mathrm{C}_{4} \mathrm{~N}_{6} \mathrm{Cu} & \text { Gef. } \\ \mathrm{Cu} & 32,50 & \mathbf{3 2 , 6 1}\end{array}$


Wenn man in eine heiße Lösung oder in eine Suspension des frisch gefällten Cuprisalzes schweflige Säure einleitet, so erhält man das Cuprosalz als farblosen, feinkrystallinischen, in Wasser fast unlöslichen Niederschlag. Beim Stehen an der Luft findet eine teilweise Rückoxydation statt.

Das Bleisalz des Dicyanamids fällt als fein krystalliner, auscheinend aus kubischen Krystallen bestehender farbloser Niederschlag auf Zusatz von Bleiacefat. Es ist in Wasser und veraünten Säuren fast unlöslich. Bei starkem Erhitzen zersetzt sich das Bleisalz unter Aufglïhen.

Das Mercurisalz erhält man als farblosen krystalli. nischen, in Wasser und verdünnten Mineralsäuren sehr schwer löslichen Niederschlag, wenn man zu einer heißen Lösung von Quecksilberoxyd in verdünnter Salpersäure Natriumdicyanamid hinzuf̈̈gt. Mit Quecksilberchlorid entsteht keine Fällung.

Mit Mercuronitrat erhält man einen auch in heibem Wasser fast unlöslichen Niederschlag des Mercurosalzes. Das trockene Salz zersetzt sich beim Erhitzen unter starkem Aufblähen.

Das Ammoniumsalz des Dicyanamids wurde in der Weise dargestellt, daß in eine ammoniakalische Lösung des Kupfersalzes so lange Schwefelwasserstoff eingeleitet wurde, bis eine abtiltrierte Probe auf Zusatz von Schwefelammonium kein Schwefelkupfer mehr abschied. Nach dem Abfiltrieren vom Schwefelkupfer wurde so lange zum Sieden erhitzt, bis die Lösung ganz farblos geworden war und sich kein Schwefel mehr ausschied. Der fein verteilte amorphe Schwefel kann durch Kochen mit etwas Papierbrei leicht in filtrierbaren Zustand gebrach $\$$ werden. Nach dem Einengen des Filtrats scheidet sich das Ammoniumsalz aus der erkaltenden Lösung in schönen großen Krystallen aus. Zur völligen Reinigung wurde das Salz aus Alkohol umkrystallisiert. Man kann es auch mit $\ddot{A}$ ther in Gestalt feiner weißer Nadeln ausfällen. 
$0,084 \mathrm{~g}$ gaben $48 \mathrm{cem}$ Stickgas bei $14^{\circ}$ und $752 \mathrm{~mm}$ Druck.

$\begin{array}{ccc} & \text { Ber. für } \mathrm{C}_{2} \mathrm{H}_{4} \mathrm{~N}_{3} & \text { Gef. } \\ \mathrm{N} & 66,67 & 66,20\end{array}$

Das Salz ist äußerst leicht in Wasser löslich, ziemlich leicht auch in Alkohol. Das analysenreine Sal\% schmilzt bei $116^{\circ}$. Erhitzt man weiter, so beginnt bei $126^{\circ}$ Zersetzung unter Ammoniakabspaltung. Das Salz färbt sich gelblich und geht anscheinend in die polymerisierte Form der freien Säure über. Bei hoher Temperatur schmilzt die Masse dann abermals anter tieferer Gelbfärbung.

Das Forrisalz des Dicyanamids liegt augenscheinlich in der roten Lösung vor, die man erhält, wenn man eine Lösung eines Dicyanamidsalzes mit einigen Tropfen Eisenchlorid versetzt. Die rote Farbe ist aber wesentlich schwächer als diejenige, die man unter gleichen Bedingungen mit einer Rhodanlösung erhält.

Das gleiche gilt auch von einer anderen ebenfalls für Rhodansalze charakteristischen Reaktion. Fügt man nämlich zu einer konz. Lösung eines Dicyanamidsalzes einige Tropfen konz. Salpetersäure, so erhält man eine deutliche Rotfärbung, die bei weiterem Zusatz von konz. Salpetersäure wieder verschwindet.

\section{Dicyanmethylamid.}

1,73 g Dicyanamidsilber und 1,4 $\mathrm{g}$ Jodmethyl werden zusammen mit $50 \mathrm{ccm}$ Benzol im zugeschmolzenen Glasrohr 10 Stunden lang auf $100^{\circ}$ erhitzt. Nach dem Öffnen der Bombenröhre wird vom gebildeten Jodsilber und dem zum großen Teil unveränderten Dicyanamidsilber abfiltriert. Aus dem Filtrat scheidet sich das Methylierungsprodnkt beim Einengen in Form quadratischer Krystalle ab, die bei $221^{\circ}$ ohne Zersetzung schmelzen.

$0,0526 \mathrm{~g}$ gaben $23,8 \mathrm{ccm}$ Stickgas bei $15^{\circ}$ und $750 \mathrm{~mm}$ Druck.

Ber. für $\mathrm{C}_{3} \mathrm{H}_{4} \mathrm{~N}_{3}$

Gef.

$\mathbf{N}$

51,85

52,01

Axanlen der Chemie 427. Baad. 
Versuche zur Darsteilung des freien Dicyanamids.

Dicyanamid hat es mit Cyanamid gemein, daß es anch aus konzentrierterer wäBriger Lösung nicht in ein indifferentes organisches Lösungsmittel übergeführt werden kann.

Daraus ergab sich folgender Versuch, Dicyanamid $\mathrm{zu}$ isolieren.

Nach D.R.P. $164724^{\text {y) }}$ gelingt es, wie wir bestätigen können, leicht, Cyanamid aus der wäßrigen Lösung des Natriumsalzes dadurch in Freiheit zu setzen, daB man die berechnete Menge Schwefelsäure in einer solchen Konzentration zusetzt, daß das sämtliche Wasser als Krystallwasser an das auskrystallisierende Natriumsulfat, gebunden ist. Das Cyanamid kann darauf mit Äther extrahiert werden. Entsprechend diesem Verfahren wurde auch Dicyanamidnatrium unter guter Kühlung mit der berechneten Menge Schwefelsäure versetzt und danach noch soviel wasserfreies Natriumsulfat zugesetzt, daß alles Wasser als Krystallwasser gebunden war. Der Krystallbrei wurde darauf mehrmals mit Äther ausgezogen. In den Äther ging ein wenig einer Verbindnng hinein, die nach ihren Eigenschaften als Dicyanamid angesprochen werden konnte. Die Lösung war aber nur kurze Zeit beständig.. Sie schied schon unmittelbar nach dem Filtrieren einen amorphen weißen Niederschlag aus. Nach dem Verdunsten des Äthers war ein geringer Teil des Rückstandes noch in Wasser mit stark saurer Reaktion löslich, die Lösung ließ aber alsbald die gleichen amorphen Flocken ausfallen, die sich immer ausscheiden, wenn man die Lösung eines Dicyanamidsalzes mit Mineralsäure versetzt.

Ein weiterer Versuch wurde in der Weise ausgeführt, daß in Alkohol suspendiertes Dicyanamidkupfer mit Schwefelwasserstoff behandelt wurde. Nach dem Ab- 
filtrieren des Schwefelkupfers wurde im Vakuum eingeengt. Es schied sich hierbei die gleiche amorphe Masse ans wie beim ersten Versuche.

\section{Leitfähigkeitsbestimmungen.}

Für die Bestimmung des Grenzwertes der Leitfähigkeit wurde die Leitfähigkeit des Natriumsalzes bei $25^{\circ}$ in den bekannten Verdünnungen gemessen und der Grenzwert durch Extrapolation nach Bredigs empirischer Regel berechnet.

Dicyanamidnatrium.

\begin{tabular}{|c|c|c|c|c|c|c|}
\hline$p$ & 32 & 64 & 128 & 256 & 512 & 1024 \\
\hline$A$ bei $25^{\circ}$ & 85,7 & 88,0 & 89,3 & 91,8 & 94,2 & 96,3 \\
\hline$t_{\infty}$ bei $25^{\circ}$ & 97,7 & 98 & 97,3 & 97,8 & 98 & $98,3\left\{\begin{array}{c}\text { inn Mittel } \\
98\end{array}\right.$ \\
\hline
\end{tabular}

Es ergibt sich darans der Grenzwert der Leitfähigkeit des freien Dicyanamids bei $25^{\circ}$ im Mittel zu 392.

Das Äquivalentleitvermögen des freien Dicyanamids wurde in der Weise bestimmt, daß za den Lösangen des Natriomdicyanamids in den üblichen Verdünnungen zanächst die äquivalente Menge titrierter Salzsäure hinzugefügt und dann sofort das elektrische Leitvermögen dieser Mischung gemessen wurde. Der gefundene Wert entspricht der Summe des Leitvermögens einer der angewandten Menge des Natriumsalzes äquivalenten Menge freien Dicyanamids und der des durch doppelte Umsetzung aus dem Natriumsalz entstandenen Natriumchlorids. Um daraus das Äquivalentleitvermögen des Dicyanamids zu berechnen, muß man also von dem gemessenen Leitvermögen der Mischung das bekannte Leit-

1) Für die Berechnung von $\mu_{\infty}$ einer Sänre bei $25^{\circ}$ ist nach Ostwald zu $\mu_{\infty}$ des Natriumsalzes die Differenz der Wanderungsgesehwindigkeiten des H-Ions $=325$ und des Na-Ions $=49,2$ zu addieren. Diese Zahl $=\mathbf{2 7 5}, 8$ ergibt für die Einheit des Leitvermögens von Quecksilber auf empirische $\mathrm{Ohm}$ umgerechnet die Zahl 294, die zu $A_{\infty}$ des Natriumsalzes zwecks Festatellung von $A_{\infty}$ der Säure addiert werden muß. 
vermögen von Natrinmchlorid der jeweiligen Verdünnung in Abzug bringen and die Differenz durch die Äquivalentkonzentration dividieren.

$$
A\left(\mathrm{C}_{2} \mathrm{~N}_{3} \mathrm{H}\right)=\frac{x\left(\mathrm{C}_{2} \mathrm{~N}_{3} \mathrm{H}+\mathrm{NaCl}\right)-x(\mathrm{NaCl})}{\eta} .
$$

$\begin{array}{rcccccc}1 / 10^{3}=v & 64 & 128 & 256 & 512 & 1024 & 2048 \\ A \text { bei } 25^{\circ} & 369 & 371 & 372 & 373 & 374 & 374\end{array}$

Dicyanamid ist demnach eine sehr starke Säure, da $A$ schon bei geringer Verdünnung sehr groß ist und durch weitere Verdünnung dem Grenzwert nur wenig näher gebracht werden kann.

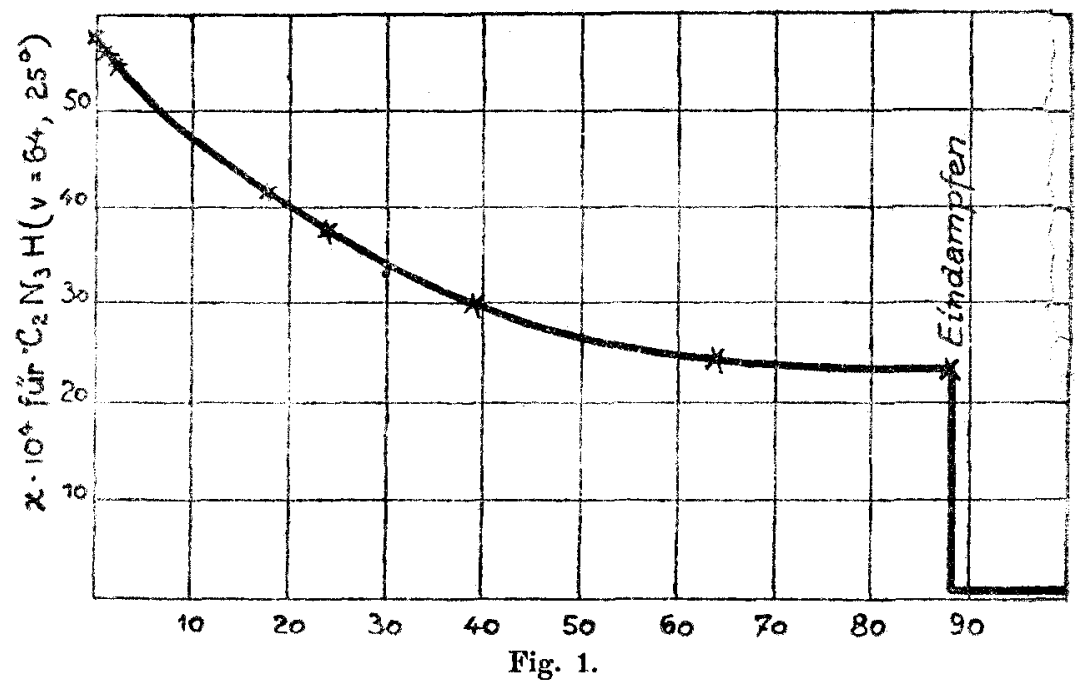

Das Leitvermögen eines in der angegebenen Weise erhaltenen Gemisches von freier Säure und Natriumchlorid zeigt nur im ersten Momente nach der Vereinigung seiner Bestandteile den angegebenen Wert. Dieser sinkt alsbald entsprechend der eintretenden Polymerisation des Dicyanamids. Aus dem Verlauf der Kurve, die für ein solches Gemisch aufgenommen wurde, geht hervor, daß der Wert des Leitvermögens bei mäßiger Verdünnung $(v=64)$ und $25^{\circ}$ sich nach anfangs schnellem. Abfall einem konstanten Wert nähert, der einem noch 
Leitrermögen besitzenden Polymerisat entspricht. Dieser stabile Zustand ist nach 64 Standen praktisch erreicht, da jetzt ein wesentliches Sinken des Leitvermögens nicht mehr beobachtet wird. Wenn man nunmehr aber die ganze Lösung auf dem Wasserbade eindampft und dann wieder durch Zusatz von Wasser anf die Anfangskonzentration bringt, so findet man nur noch das Leitvermögen des Natriumchlorids. (Nach Ostwald ist $* .10^{4}$ für $\mathrm{NaCl}\left(25^{\circ}, v=64\right) 18,27$, gefunden warde $x \cdot 10^{4}=18,63$ ). Das Dicyanamid wandelt sich durch das Findampfen in ein amorphes Palver nm, das praktisch unlöslich und nichtleitend geworden ist.

\section{Anlagerungsreaktionen des Dicyanamids.}

Biuret.

Während man beim Erhitzen der wãßrigen Lösung von Natriumdicyanamid mit der berechneten Menge verdünnter Salzsäure nur ein amorphes Polymerisationsprodukt erhält, wird glatte Überführung in Biuret unter Anlagerung von $2 \mathrm{Mol}$. Wasser erzielt, wenn man das Salz in wenig Wasser löst und mit dem doppelten Volumen konzentrierter Salzsäure etwa 5 Minuten lang zam Sieden erhitzt. Es scheiden sich farblose Nadeln aus, die nach dem Umkrystallisieren den Schmelzp. $190^{\circ}$ besitzen und mit Kapfersulfat und Natronlange die Biuretreaktion geben.

\section{Cyanguanidin (Dicyandiamid) und Biguanid.}

Das Kupfersalz des Dicyanamids wurde in überschüssigem konzentrierten Ammoniak gelöst und die tiefblane Lösung im Bombenrohr während 12 Stnnden auf $150^{\circ}$ erhitzt. Nach dem Öffnen der Röhre waren in dem von etwas unveränderter ammoniakalischer Kupferlösung des Dicyanamids abfiltrierten Rückstande deutlich zwei Krystallformen wahrnehmbar, große, weiße blättrige Krystalle und kleine rote Nadeln. In einem Versuche waren die weißen Krystalle zum Teil so groß aus- 
gebildet, daB eine mechanische Trennung von den roten Nadeln leicht durchgeführt werden konnte. Sie erwiesen sich nach dem Umkrystallisieren aus wenig Wasser als reines Cyanguanidin (Dicyandiamid) rom Schmelzp. 205". Bei weiteren in gleicher Weise angestellten Versuchen gelang es nicht mehr, das Cyanguanidin als Zwischenkörper za fassen, da alles Kupfersalz in die charakteristischen roten Nadeln von Biguanidknpfer verwandelt wurde. Der Übergang von Cyanguanidin in jene Verbindung unter den Versuchsbedingungen ist bekannt. ${ }^{1}$ ?

\section{O-Ähylcyanisoharnstoff.}

a) Direkte Darstellung aus Cyanamid und Bromeyan.

$\mathrm{Zu}$ einer Lösung von 4,2 $\mathrm{g}$ Cyanamid in ca. $100 \mathrm{ccm}$ absolutem Alkohol wurde zunächst eine ebenfalls alkoholische Liosung von Natriumalkoholat aus 4,6 g Natrium hinzugefügt. $Z a$ dieser Lösung warde dann unter Kühlung eine solche von Bromcyan in geringem Überschub über die Theorie $(10,6 \mathrm{~g})$ zugetropft und darauf einige Stunden stehen gelassen. Dann wurde vom ausgeschiedenen Natriumbromid abfiltriert und auf dem Wasserbade eingeengt. Es scheiden sich nacheinander zwei verschiedene Natriumsalze aus, die sich aber durch ihre verschiedene Löslichkeit voneinander trennen lassen. Mehrere Versuche, die sich in der Versuchsanordnung anscheinend nicht wesentlich unterschieden, führten insofern zu verschiedenen Ergebnissen, als in einem Falle nur Natriumdicyanamid, in einem anderen das Natriumsalz des 0 Äthylcyanisoharnstoffs isoliert wurde. Ersteres Salz ist das stabilere, in das das Cyanisoharnstoffäthersalz übergeht. Das geschieht, wenn auch sehr langsaru, schon bei Zimmertemperatur, wenigstens enthielt ein Präparatengläschen dieses Salzes nach mehreren Jahren

1) Huth, Monatsh f. Chem. 1, 88 (1880). 
nur noch Natriumdicyanamid. Das Natriumsalz des O-Äthylcyanisoharnstoffs krystallisiert in farblosen Nädelchen und besitzt in wäßriger Lösung schwach alkalische Reaktion.

$0,1524 \mathrm{~g}$ gaben $0,1893 \mathrm{CO}_{2}$ und $0,0553 \mathrm{H}_{2} \mathrm{O}$.

$0,1427 \mathrm{~g}$ " $0,1797 \mathrm{CO}_{2}, 0,0548 \mathrm{H}_{2} \mathrm{O}$.

$0,0931 \mathrm{~g} \quad 24,8$ ccm Stickgas bei $15^{\circ}$ und $768 \mathrm{~mm}$ Druck.

Ber. für $\mathrm{C}_{4} \mathrm{H}_{8} \mathrm{ON}_{3} \mathrm{Na}$

Gef.

$\begin{array}{rrrr}\mathrm{C} & 35,53 & 34,10 & 34,34 \\ \mathrm{H} & 4,47 & 4,09 & 4,30 \\ \mathrm{~N} & 31,12 & 31,91 & -\end{array}$

Der freie Cyanisoharnstoffäther wird aus dem $\mathrm{Na-}$ triumsalz durch Säuren in Freiheit gesetzt, man erhält ihn daher auch, wenn man Bromcyan im Überschub auf die alkoholische Lösung von Cyanamid und Natriumalkoholat einwirken läßt, da bei seiner allmählich eintretenden Zersetzung Bromwasserstoff gebildet wird. Man kann ihn jedoch auch schon erhalten, wenn man eine schwache Säure, wie Essigsäure, in berechneter Menge zur Lösung des Natriumsalzes hinzusetzt und eindampft. Aus dem Rückstande wird die Verbindung lejeht durch Äther aufgenommen. O-Äthylcyanisoharnstoff ist eine ganz beständige, in wäßriger Lösung völlig neutral reagierende Verbindung. Sie ist in den meisten organischen Iösungsmitteln sehr leicht löslich. Für die Analyse wurde die Verbindung aus Benzol amkrystallisiert, worin sie verhăltuismäßig schwer löslich ist. Man erhält sie daraus in derben Nadeln oder Prismen vom Schmelzp. $119^{\circ}$.

$$
\begin{aligned}
& 0,1615 \mathrm{~g} \text { gaben } 0,2521 \mathrm{CO}_{2} \text { und } 0,0865 \mathrm{H}_{9} \mathrm{O} \text {. } \\
& 0,1612 \mathrm{~g}, \quad 0,2510 \mathrm{CO}_{2} \quad, \quad 0,914 \mathrm{H}_{2} \mathrm{O} \text {. } \\
& 0,1195 \mathrm{~g} \quad, \quad 38,3 \mathrm{ccm} \text { Stickgas bei } 17^{\circ} \text { und } 760 \mathrm{~mm} \text { Druck. } \\
& 0,0931 \mathrm{~g} \quad 29,2 \mathrm{ccm} \quad \text { " } 15^{\circ}, 770 \mathrm{~mm} \text {, } \\
& \text { Ber. für } \mathrm{C}_{4} \mathrm{H}_{7} \mathrm{ON}_{9}
\end{aligned}
$$

$\begin{array}{rrrr}\mathrm{C} & 42,44 & 42,57 & 42,47 \\ \mathrm{H} & 6,24 & 5,99 & 6,34 \\ \mathrm{~N} & 37,17 & 37,73 & 37,66\end{array}$


Darstellung durch Anlagerung von Alkohol an Dicyanamid.

$\mathrm{Zu} 9 \mathrm{~g}(1 / 10 \mathrm{Mol})$ Natriumdicyanamid warde in absolut alkoholischer Lösung die äquivalente Menge titrierter alkoholischer Salzsäure hinzugefügt. Nach mehrstündigem Stehen wurde eingedampft und das entstandene Anlage-

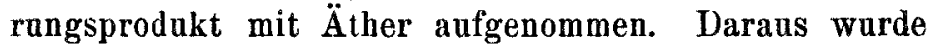
etwa $1 \mathrm{~g}$ der Verbindung vom Schmelzp. $119^{\circ}$ isoliert. Der Rest des Dicyanamids hatte sich in eine amorphe anlösliche Sabstanz verwandelt.

\section{o. Atthylisobiuret.}

$9 \mathrm{~g}$ Natriumdicyanamid wurden in $50 \mathrm{ccm}$ absolutem Alkohol suspendiert and überschüssiger Chlorwasserstoff anter Kühlung eingeleitet. Das Natriumdicyanamid ging in Lösung, während sich zunächst Natriumchlorid und im Verlauf von einer Stunde auch das Hydrochlorid des O- ̈̈thylisobiurets als dicker Krystallbrei ausschied. Unter dem Mikroskop waren außer den Würfeln des Natriumchlorids nur die Nadeln des entstandenen Hydrochlorids u erkennen, und in Wasser löste sich das Reaktionsprodukt klar und rückstand]os anf. Ein amorphes Polymerisationsprodukt entsteht also unter diesen Bedingungen nicht. Das natriumchloridhaltige Rohprodukt wurde abgesaugt. Um daraus die freie Base zu isolieren, wurde nach dem für 0 -Alkylisoharnstoffe angegebenen Verfahren ${ }^{1}$ ) das Hydrochlorid mit wenig Wasser und pulverförmigem Ätzkali versetzt und mit viel Äther ausgeschüttelt. Beim Einengen des Äthers schied sich die freie Base in würfelähnlichen Kryställchen aus. Nach ¿weimaligem Umkrystallisieren aus Alkohol liegt der Schmelzp. bei $1 \% 9^{\circ}$. Die Verbindung ist in den meisten organischen Lösungsmitteln leicht löslich, schwerer in Benzol. In wäBriger Lösung zeigt sie schwach alkalische

1) Stieglitz u. Me Kee, Ber. d. d. chem. Ges. 33, 1517 (1900). 
Reaktion. Durch Zusatz von Salzsänre zu der Lơsung in Alkohol wird das schwer lösliche Chlorhydrat wieder ausgefällt.

$0,1856 \mathrm{~g}$ gaben $0,2532 \mathrm{CO}_{2}$ und $0,1181 \mathrm{H}_{2} \mathrm{O}$.

$0,1230 \mathrm{~g}, \quad 34,6 \mathrm{cem}$ Stickgas bei $18^{\circ}$ und $760 \mathrm{~mm}$ Druck.

$\begin{array}{ccc} & \text { Ber. für } \mathrm{C}_{4} \mathrm{H}_{9} \mathrm{O}_{4} \mathrm{~N}_{8} & \text { Gef. } \\ \mathrm{C} & 36,64 & 37,21 \\ \mathrm{H} & 6,87 & 7,13 \\ \mathrm{~N} & 32,06 & 33,0\end{array}$

Um sicher za gehen, daß das sich zanächst ausscheidende Reaktionsprodukt die Zusammensetzung eines Hydrochlorids des O-Äthylisobiurets hat, eine Abspaltung von Chloräthyl nicht erst bei der Aufarbeitung zur freien Base aus zanächst entstandenem Dihydrochlorid stattfindet, wurde eine Elementaranalyse des Rohprodukts ausgeführt, indem von der abgewogenen Substanz das im Schiffchen zurückbleibende Natriumclslurid abgezogen wurde.

$0,3086 \mathrm{~g}$ gaben $0,3620 \mathrm{CO}_{2}$ und $0,1680 \mathrm{H}_{2} \mathrm{O}$.

$\begin{array}{ccc} & \text { Ber. für } \mathrm{C}_{4} \mathrm{H}_{10} \mathrm{O}_{2} \mathrm{~N}_{3} \mathrm{Cl} & \text { Gef. } \\ \mathrm{C} & 29,22 & 31,99 \\ \mathrm{H} & 5,40 & 5,68\end{array}$

Das sich bei der Einwirkung überschüssiger alkoholischer Salzsäure ansscheidende Hydrochlorid ist demnach zweifellos das Monohydrochlorid des 0-Äthylisobinrets.

\section{Cyanharnstoff.}

Die Bildung dieser Verbindang in Form ihres Natrinmsalzes wurde beobachtet bei dem Versuche, ein als Reaktionsprodukt zwischen Dinatriumeyanamid und Brom vermutetes Natriumbromcyanamid mit Natriumcyanid umzusetzen.

$\mathrm{Zu}$ einer Lösung von $8,6 \mathrm{~g}$ technischem Natriumcyanamid in wenig Wasser wurde unter Kühlung $16 \mathrm{~g}$ Brom hinzugetropft. Gegen Ende der Zugabe des Broms schied sich ein in Nadeln krystallisierender Körper ab, der sich aber beim Versuche, ihn zu isolieren, zersetzte. Auf Zusatz einer Lösung von $5 \mathrm{~g}$ Natriumcyanid ging 
der zunächst entstandene Niederschlag wieder in Lơsung. beim Stehen schieden sich dann aber nadelförmige Krystalle eines Natrinmsalzes ab, deren Lösung mit Silbernitratlösung ein farbloses Silbersalz ausfallen ließ.

$0,1744 \mathrm{~g}$ gaben $32,4 \mathrm{ccm}$ Stickgas bei $18^{\circ}$ und $738 \mathrm{~mm}$ Druck.

$0,3512 \mathrm{~g} \# \quad 0,2609 \mathrm{AgCl}$.

$\begin{array}{lcc} & \text { Ber. für. } \mathrm{C}_{2} \mathrm{H}_{2} \mathrm{ON}_{3} \mathrm{Ag} & \text { Gef. } \\ \mathrm{N} & 21,87 & 21,20 \\ \mathrm{Ag} & 56,25 & 55,90\end{array}$

Den Direktionen der Farbenfabriken vorm. Fr. Bayer \& Co. in Leverkasen und der Deatschen Gold- and Silberscheide-Anstalt vorm. Rößler in Frankfurt a. $\mathbf{M}$. sagen wir für überlassene Präparate unsern besten Dank.

\section{Ober Tricyanmelamin; von $W$. Madelung und E. Kern.}

Wenn man Dicyanamidnatrium auf höhere Temperatur erhitzt, so verwandelt es sich in seine trimere Verbindung. Diese Verbindung steht als Cyanurderivat zur Ausgangsverbindung zweifellos im gleichen Verhältnis. wie das Melamin (Cyanuramid) zum Cyanamid. Wie nun für das Melamin die Aufstellung mehrerer Formeln möglich ist, die aber desmotropen Charakter haben und deren Grenzfälle das normale Melamin und das Isomelamin sind, so gibt es auch für das Tricyanmelamin mehrere Formulierungsmöglichkeiten, die sich von den desmotropen Melaminformeln ableiten lassen, aber selber nicht mehr desmotropen Verbindungen entsprechen.<smiles>N#CNc1nc(NC#N)nc(NC#N)n1</smiles>

exo-Tricyanmelamin $(2,4,6-T$ Tris-[cyansmino-] 1,3,5-triazin)<smiles>CN1C(=N)C(=N)N(C)C(=N)C1=N</smiles>

eso-'Tricyanisomelamin (1,3,5-Tricyan- $-4,4,6$-trimino1,3,5-triazin-hexahydrid.) 